\title{
HARMONIC WAVE PROPAGATION IN AN INFINITE VISCOELASTIC MEDIUM WITH A PERIODIC ARRAY OF CYIINDRICAL ELASTIC FIBERS $\dagger$
}

\author{
T.-C. MAs, R. A. ScotT AND W. H. YANG \\ Department of Mechanical Engineering and Applied Mechanics, University of Michigan, Ann Arbor, \\ Michigan 48109, U.S.A.
}

(Received 29 May 1979, and in revised form 28 September 1979)

\begin{abstract}
The work involves the propagation of plane harmonic waves in an infinite isotropic medium in which a doubly periodic array of cylindrical fibers is embedded. The direction of propagation is perpendicular to the fibers and the matrix material is taken to be viscoelastic in shear, modeled through hereditary integrals. A finite element method based on Galerkin's technique is employed, which leads to a non-linear eigenvalue problem. An iterative scheme is used to obtain two modes of dispersion, for both real and imaginary wave numbers, for a specific composite.
\end{abstract}

\section{INTRODUCTION}

Because of their attractive strength to weight properties, composite materials continue to receive considerable attention in the mechanics literature, as regards both statics and dynamics (see reference [1]). A common assumption in addressing wave propagation has been that both matrix and filler materials behave elastically and recent reviews of such work can be found in references [2-4]. In many situations however, the matrix material behaves viscoelastically, and work along these lines has started to emerge. Stern, Bedford and Yew [5] used an effective modulus theory to analyze one-dimensional wave propagation in a laminated medium consisting of alternating layers of elastic and viscoelastic materials. Demiray and Eringen [6] developed a two-dimensional lattice dynamics model to study harmonic wave propagation in a fibrous material, in which they assumed that the viscoelastic matrix carries the shear and the fibers carry the tensile forces. Mukherjee and Lee $[7,8]$ used finite difference and variational methods to examine one-dimensional wave propagation in a viscoelastic laminated composite. Sutherland [9] presented some experimental studies on dispersion in fiber reinforced viscoelastic materials. The correspondence principle could be used to obtain information where analytical results are available, such as is the case for some approximate theories. However, such results are not available when linear "exact" theories are used, such as here.

The present paper is an extension of the finite element work of Golub, Jenning and Yang [10] on harmonic wave propagation in an infinite elastic medium reinforced by a periodic array of cylindrical elastic fibers, the direction of propagation being perpendicular to the fibers. Here the matrix material is taken to be viscoelastic in shear, but to behave elastically under hydrostatic load, a not uncommon assumption (see reference [11]). Such a problem

†This work is part of a Ph.D. Dissertation in Applied Mechanics by the first author. ¥Currently at Exxon Production Research Company, Houston, Texas 77001, U.S.A. 
is intractable analytically and difficult numerically. One of the goals of the present study was to see if modern developments in computation could lead to useful results within the framework of a limited budget. It was found that the technique employed, namely, a finite element scheme based on Galerkin's method together with an iterative matrix solution technique, was indeed a powerful tool for attacking this class of problems.

\section{EQUATIONS OF MOTION}

The model employed consists of a doubly periodic array of circular cylindrical fibers embedded in an infinite matrix, the direction of wave propagation being perpendicular to the fibers (see Figure 1). Each constituent is taken to be homogeneous and isotropic. Under these circumstances, a plane strain state holds and for harmonic wave motion, the following forms are assumed, in standard notation:

$$
\begin{gathered}
\bar{\sigma}_{x x}(x, y, t)=\sigma_{x x}(x, y) \mathrm{e}^{\mathrm{i} \omega t}, \bar{\sigma}_{y y}(x, y, t)=\sigma_{y y}(x, y) \mathrm{e}^{\mathrm{i} \omega t}, \bar{\sigma}_{x y}(x, y, t)=\sigma_{x y}(x, y) \mathrm{e}^{\mathrm{i} \omega t}, \\
\bar{u}(x, y, t)=u(x, y) \mathrm{e}^{\mathrm{i} \omega t}, \bar{v}(x, y, t)=v(x, y) \mathrm{e}^{\mathrm{i} \omega t} .
\end{gathered}
$$

The problem being examined is one of forced motion, with a remote source of forcing, so that $\omega$ can be regarded as real. Introducing the hydrostatic stress,

$$
\sigma=\frac{1}{3}\left(\sigma_{x x}+\sigma_{y y}+\sigma_{z z}\right)
$$

and deviatoric stresses,

$$
\begin{gathered}
\sigma_{x x}^{\prime}=\sigma_{x x}-\sigma=\frac{1}{3}\left(2 \sigma_{x x}-\sigma_{y y}-\sigma_{z z}\right), \sigma_{y y}^{\prime}=\sigma_{y y}-\sigma=\frac{1}{3}\left(2 \sigma_{y y}-\sigma_{z z}-\sigma_{x x}\right), \\
\sigma_{z z}^{\prime}=\sigma_{z z}-\sigma=\frac{1}{3}\left(2 \sigma_{z z}-\sigma_{x x}-\sigma_{y y}\right), \sigma_{y x}^{\prime}=\sigma_{x y}
\end{gathered}
$$

gives, in the absence of body forces, the equations of motion for either constituent:

$$
\frac{\partial}{\partial x}\left(\sigma+\sigma_{x x}^{\prime}\right)+\frac{\partial}{\partial y}\left(\sigma_{x y}^{\prime}\right)+\rho \omega^{2} u=0, \frac{\partial}{\partial x}\left(\sigma_{x y}^{\prime}\right)+\frac{\partial}{\partial y}\left(\sigma+\sigma_{y y}^{\prime}\right)+\rho \omega^{2} v=0 .
$$

Attention will now be focused temporarily on the matrix material. Making the common assumption that the behavior is elastic under hydrostatic stress, one gets

$$
\sigma=e K \varepsilon,
$$

where $K$ is the bulk modulus and $\varepsilon$ is the dilatational strain defined by

$$
\varepsilon=\frac{1}{3}\left(c_{x x}+\varepsilon_{y y}\right) \text {. }
$$

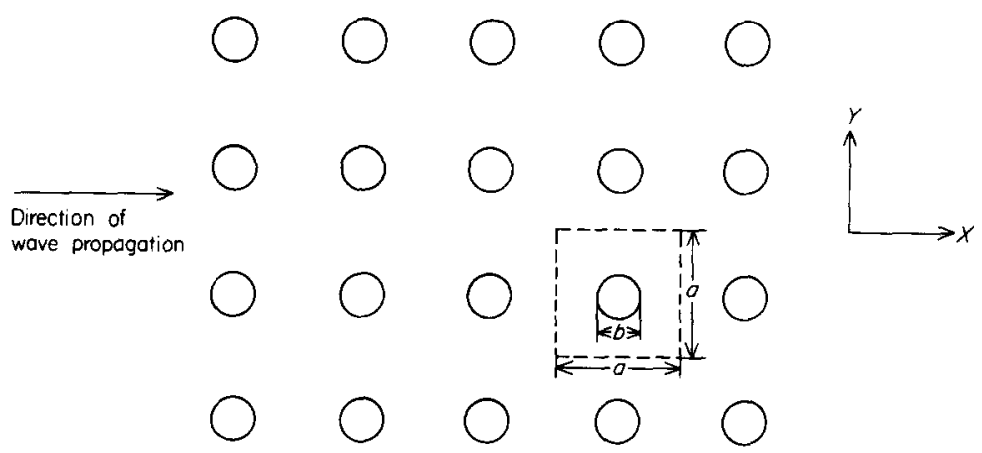

Figure 1. Material geometry. 
For an isotropic linear viscoelastic material, the deviatoric stresses are given by

$$
\bar{\sigma}_{i j}^{\prime}(x, y, t)=\int_{-\infty}^{t} Y(t-\tau) \mathrm{d} \bar{\varepsilon}_{i j}^{\prime}(x, y, \tau), i=x, y \cdot j=x, y,
$$

where $Y$ is the relaxation function and the distortion strains $\tilde{\varepsilon}_{i j}^{\prime}$ are defined by

$$
\begin{gathered}
\bar{\varepsilon}_{x x}^{\prime}=\bar{\varepsilon}_{x x}-\bar{\varepsilon}=\bar{\varepsilon}_{x x}-\frac{1}{3}\left(\bar{\varepsilon}_{x x}+\bar{\varepsilon}_{y y}\right)=\frac{1}{3}\left(2 \bar{\varepsilon}_{x x}-\bar{\varepsilon}_{y y}\right), \\
\bar{\varepsilon}_{y y}^{\prime}=\bar{\varepsilon}_{y y}-\bar{\varepsilon}=\bar{\varepsilon}_{y y}-\frac{1}{3}\left(\bar{\varepsilon}_{x x}+\bar{\varepsilon}_{y y}\right)=\frac{1}{3}\left(2 \bar{\varepsilon}_{y y}-\bar{\varepsilon}_{x x}\right), \\
\bar{\varepsilon}_{x y}^{\prime}=\bar{\varepsilon}_{x y^{\prime}}
\end{gathered}
$$

The strain displacement relations are

$$
\bar{\varepsilon}_{x x}=\partial \bar{u} / \partial x, \bar{\varepsilon}_{y y}=\partial \bar{v} / \partial y, \bar{\varepsilon}_{x y}=\frac{1}{2}(\partial \bar{u} / \partial y+\partial \bar{v} / \partial x),
$$

and using them, logether with equations (1)-(4), (8) and (10), equations (9) can be written as

$$
\sigma_{i j}^{\prime}(x, y)=2 G(\omega) \varepsilon_{i j}^{\prime}(x, y), i=x, y, j=x, y,
$$

where

$$
2 G(\omega)=\mathrm{i} \omega \int_{0}^{\infty} y(\eta) \mathrm{e}^{-\mathrm{i} \omega \eta} \mathrm{d} \eta
$$

and is called the complex modulus. Using equations (7), (10), (11) and (12) equations (5) and (6) become

$$
\begin{aligned}
& G\left(\partial^{2} u / \partial y^{2}+\partial^{2} v / \partial x \partial y\right)+\underline{\alpha} \partial^{2} u / \partial x^{2}+\beta \partial^{2} v / \partial x \partial y+\rho \omega^{2} u=0, \\
& G\left(\partial^{2} u / \partial x \partial y+\partial^{2} v / \partial x^{2}\right)+\beta \partial^{2} u / \partial x \partial y+\underline{\alpha} \partial^{2} v / \partial y^{2}+\rho \omega^{2} v=0,
\end{aligned}
$$

where for the viscoelastic material

$$
\underline{G}=G_{v}(\omega), \underline{\alpha}=K_{v}+\frac{4}{3} G_{v}(\omega), \underline{Q}=K_{v}-\frac{2}{3} G_{v}(\omega),
$$

and for the elastic material

$$
\underline{G}=G_{e}, \underline{\alpha}=K_{e}+\frac{4}{3} G_{e}, \underline{\beta}=K_{e}-\frac{2}{3} G_{e} .
$$

From Floquet theory, the following quasi-periodicity conditions must be satisfied:

$$
\begin{aligned}
u\left(\frac{a}{2}, y\right) & =\mathrm{e}^{\mathrm{i} q a} u\left(-\frac{a}{2}, y\right), v\left(\frac{a}{2}, y\right)=\mathrm{e}^{\mathrm{i} q a} v\left(-\frac{a}{2}, y\right), \\
\frac{\partial}{\partial x} u\left(\frac{a}{2}, y\right) & =\mathrm{e}^{\mathrm{i} q a} \frac{\partial}{\partial x} u\left(-\frac{a}{2}, y\right), \frac{\partial}{\partial x} v\left(\frac{a}{2}, y\right)=\mathrm{e}^{\mathrm{i} q a} \frac{\partial}{\partial x} v\left(-\frac{a}{2}, y\right),
\end{aligned}
$$

where $q$ denotes wavenumber. In addition, symmetry requires that the normal displacements and shear stresses are zero on the half-cell boundaries at $y=0$ and $a / 2$ :

$$
v(x, 0)=v\left(x, \frac{a}{2}\right)=0, \frac{\partial u}{\partial y}(x, 0)=\frac{\partial u}{\partial y}\left(x, \frac{a}{2}\right)=0 .
$$

Finally, perfect bonding is assumed at the matrix-fiber interface, so that the displacements and stresses are continuous there.

\section{NUMERICAL PROCEDURE}

Since energy methods are not convenient for viscoelastic media, Galerkin's technique (see reference [12]) is used here. Applying it to equations (14) and integrating by parts 
yields

$$
\begin{array}{r}
-\iint_{\Omega}\left\{\underline{G}\left(\frac{\partial u}{\partial y}+\frac{\partial v}{\partial x}\right) \frac{\partial \phi}{\partial y}+\left(\underline{\alpha} \frac{\partial u}{\partial x}+\underline{\beta} \frac{\partial v}{\partial y}\right) \frac{\partial \phi}{\partial x}\right\} \mathrm{d} \Omega+\iint_{\Omega} \rho \omega^{2} u \phi \mathrm{d} \Omega+ \\
\int_{\partial \Omega}\left\{\underline{G}\left(\frac{\partial u}{\partial y}+\frac{\partial v}{\partial x}\right) \sin \bar{\alpha}+\left(\underline{\alpha} \frac{\partial u}{\partial x}+\underline{\beta} \frac{\partial v}{\partial y}\right) \cos \bar{\alpha}\right\} \phi \mathrm{d} s=0 \\
-\iint_{\Omega}\left\{\underline{G}\left(\frac{\partial u}{\partial y}+\frac{\partial v}{\partial x}\right) \frac{\partial \phi}{\partial x}+\left(\underline{\beta} \frac{\partial u}{\partial x}+\underline{\alpha} \frac{\partial v}{\partial y}\right) \frac{\partial \phi}{\partial y}\right\} \mathrm{d} \Omega+\iint_{\Omega} \rho \omega^{2} v \phi \mathrm{d} \Omega+ \\
\int_{\partial \Omega}\left\{G\left(\frac{\partial u}{\partial x}+\frac{\partial v}{\partial x}\right) \cos \bar{\alpha}+\left(\beta \frac{\partial u}{\partial x}+\underline{\alpha} \frac{\partial v}{\partial y}\right) \sin \bar{\alpha}\right\} \phi \mathrm{d} s=0
\end{array}
$$

where $\Omega$ is the domain of interest and $\bar{\alpha}$ is the angle between the normal vector to $\partial \Omega$ and the $x$-axis. Equations (17) must hold for all smooth functions $\phi$. The finite element method will now be employed.

The domain $\Omega$ in equations (17) is divided into $m$ triangular subdomains in each of which $u, v$ are approximated by a combination of three linear basis functions $\phi_{j}$ :

$$
u(x, y)=\sum_{j=1}^{3} u_{j} \phi_{j}(x, y), v(x, y)=\sum_{j=1}^{3} v_{j} \phi_{j}(x, y) .
$$

Here $\phi_{j}$ are taken to be linear functions over each triangular subdomain and to satisfy $\phi_{j}\left(x_{i}, y_{i}\right)=\delta_{i j}$, where $\delta_{i j}$ denotes the Kronecker delta and $\left(x_{i}, y_{i}\right)$ are the co-ordinates of a node. Substituting equations (18) into equations (17) and carrying out the integrations, the details of which can be found in reference [13], yields the system of homogeneous equations in the variables $\left\{u_{j}, v_{j} \mid j=1, n\right\}$, in matrix notation,

$$
[K+S+M] u=0,
$$

where $u^{t}=\left(u_{1}, v_{1}, \ldots, u_{n}, v_{n}\right), K$ and $S$ are viscoelastic stiffness matrices, with $S$ containing the boundary conditions $(16)$ and $M$ is the mass matrix. A difficult feature of the present work is that all of the matrices are functions of $\omega$, so that a non-linear eigenvalue problem arises.

The quasi-periodicity conditions (15) are handled as follows. In the numerical work a column is added outside the edge CD, the extent of the original discretization. This is

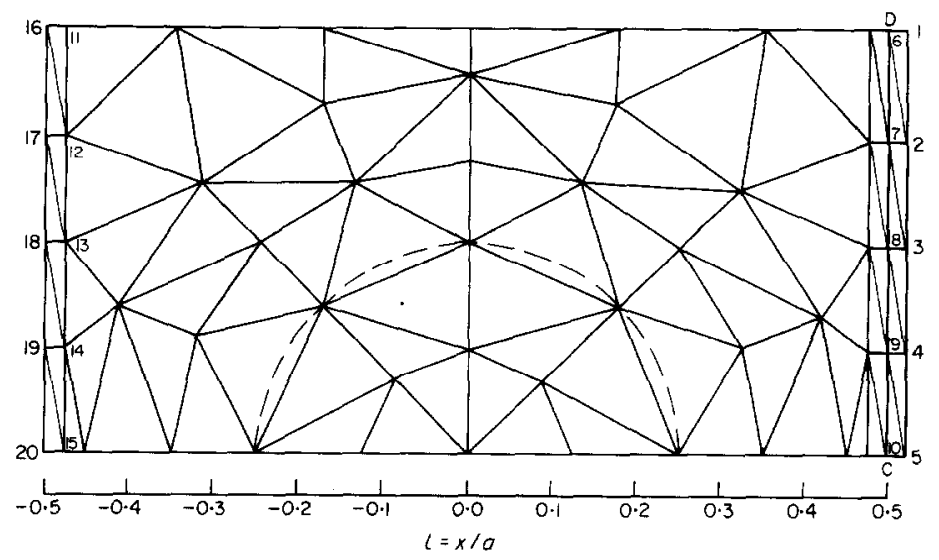

Figure 2. Dimensionless half cell and its finite element mesh configuration for the fiber reinforced, viscoelastic medium. The dashed curve indicates the matrix-fiber interface. 
illustrated by an example in Figure 2 . In the matrix operations it is required that

$$
u_{m}=u_{m^{\prime}} \mathrm{e}^{\mathrm{i} q a}, u_{n}=u_{n^{\prime}} \mathrm{e}^{\mathrm{i} q a}, v_{m}=v_{m^{\prime}} \mathrm{e}^{\mathrm{i} q a}, v_{n}=v_{n^{\prime}} \mathrm{e}^{\mathrm{i} q a}
$$

for the number pairs

$$
\begin{aligned}
& \left(m, m^{\prime}\right)=(1,11),(2,12),(3,13),(4,14),(5,15), \\
& \left(n, n^{\prime}\right)=(6,16),(7,17),(8,18),(9,19),(10,20) .
\end{aligned}
$$

Equations (20) guarantee the satisfaction of the first two conditions in equations (15). The derivative conditions in equations (15) are satisfied as follows:

$$
\left.\frac{\partial u}{\partial x}\right|_{6} \approx\left(u_{1}-u_{6}\right) / l=\left(u_{11}-u_{16}\right) \mathrm{e}^{\mathrm{i} q a} /\left.l \approx \frac{\partial u}{\partial x}\right|_{16} \mathrm{e}^{\mathrm{i} q a}
$$

where $l$ is the distance between nodal points 1 and 6 and 11 and $16 . \partial v / \partial x$ is handled similarly. The perfect bonding condition is handled by treating those interface nodal points as interior points in the finite element formulation, thereby automatically satisfying continuity of displacements.

The final form of the algebraic eigenvalue problem may be written as

$$
A(\omega, q) \psi=0 \text {, }
$$

where $A$ and $\psi$ are complex. On letting $A=A_{r}+\mathrm{i} A_{i}, \psi=\psi_{r}+\mathrm{i} \psi_{i}$, equation (23) becomes the real algebraic system

$$
N(\omega, q) \xi=0
$$

where

$$
N=\left[\begin{array}{rr}
A_{r} & -A_{i} \\
A_{i} & A_{r}
\end{array}\right], \xi=\left(\begin{array}{l}
\psi_{r} \\
\psi_{i}
\end{array}\right)
$$

Although the matrix and vector in equation (24) are real, the eigenvalues could be complex. For steady, forced oscillations, only the real eigenvalues $\omega$ are sought. These in general correspond to complex wavenumbers $q$, which implies spatial decay of the waves.

The secant iterative method is used to solve the roots of the scalar function $f(\omega)=$ $\operatorname{det}(N(\omega))$, which can be evaluated by using the result

$$
\operatorname{det}(N(\omega))=\left(\operatorname{det} \mathrm{P}^{t}\right)(\operatorname{det} L)(\operatorname{det} U)= \pm \prod_{i=1}^{n} U_{i j}
$$

where $P^{t} L U$ is the $L U$ decomposition of the matrix $N$ and $U_{i i}$ are the diagonal elements of $U$. In the secant method successive approximations to $\omega$ are calculated by

$$
\omega_{k+2}=\frac{\omega_{k+1} f\left(\omega_{k}\right)-\omega_{k} f\left(\omega_{k+1}\right)}{f\left(\omega_{k}\right)-f\left(\omega_{k+1}\right)}, k=0,1,2, \ldots,
$$

where $\omega_{0}, \omega_{1}$ are two initial estimates of a root. Equation (26) is used to check whether the true eigenvalue has been overshot.

\section{NUMERICAL RESULTS}

The fiber material is taken to be the aluminum alloy 2024-T4 with properties $K_{e}=$ $0.696 \times 10^{11} \mathrm{~N} / \mathrm{m}^{2}, G_{e}=0.276 \times 10^{11} \mathrm{~N} / \mathrm{m}^{2}$ and $\rho_{e}=0.277 \times 10^{4} \mathrm{~kg} / \mathrm{m}^{3}$. The matrix material is taken to be a natural vulcanized rubber with properties $K_{v}=3.0 \times 10^{9} \mathrm{~N} / \mathrm{m}^{2}$ 
TABLE 1

Complex modulus for natural, vulcanized rubber

\begin{tabular}{|c|c|c|}
\hline $\log _{10} \omega$ & $\begin{array}{c}G^{I} \\
\left(N / m^{2}\right)\end{array}$ & $\begin{array}{c}G^{I I} \\
\left(N / m^{2}\right)\end{array}$ \\
\hline$-4 \cdot 0$ & $4.68 \times 10^{5}$ & $1.86 \times 10^{4}$ \\
\hline$-3 \cdot 5$ & $4 \cdot 79$ & 1.91 \\
\hline$-3 \cdot 0$ & $4 \cdot 79$ & 1.91 \\
\hline$-2 \cdot 5$ & $4 \cdot 90$ & 1.91 \\
\hline$-2 \cdot 0$ & $5 \cdot 13$ & 1.91 \\
\hline-1.5 & $5 \cdot 25$ & 1.95 \\
\hline$-1 \cdot 0$ & $5 \cdot 37$ & $2 \cdot 14$ \\
\hline-0.5 & $5 \cdot 50$ & 2.35 \\
\hline 0.0 & $5 \cdot 76$ & 2.63 \\
\hline 0.5 & 5.89 & 3.02 \\
\hline $1 \cdot 0$ & $6 \cdot 17$ & 3.39 \\
\hline 1.5 & 6.46 & $4 \cdot 37$ \\
\hline $2 \cdot 0$ & 7.08 & $6 \cdot 17$ \\
\hline 2.5 & 7.77 & 11.5 \\
\hline $3 \cdot 0$ & 8.51 & $22 \cdot 4$ \\
\hline $3 \cdot 5$ & $10 \cdot 3$ & 47.9 \\
\hline-4.0 & 13.5 & 95.5 \\
\hline $4 \cdot 5$ & $19 \cdot 1$ & $214 \cdot 0$ \\
\hline $5 \cdot 0$ & $28 \cdot 2$ & $502 \cdot 0$ \\
\hline $5 \cdot 5$ & $50 \cdot 2$ & $1290 \cdot 0$ \\
\hline 6.0 & 107.0 & 3090.0 \\
\hline 6.5 & $339 \cdot 0$ & $7590 \cdot 0$ \\
\hline $7 \cdot 0$ & $1180 \cdot 0$ & $15200 \cdot 0$ \\
\hline $7 \cdot 5$ & 3390.0 & $33900 \cdot 0$ \\
\hline 8.0 & $6610 \cdot 0$ & $26300 \cdot 0$ \\
\hline $8 \cdot 5$ & $9330 \cdot 0$ & $23500 \cdot 0$ \\
\hline $9 \cdot 0$ & $11800 \cdot 0$ & $18700 \cdot 0$ \\
\hline 9.5 & $13800 \cdot 0$ & $13800 \cdot 0$ \\
\hline $10 \cdot 0$ & $14100 \cdot 0$ & $9550 \cdot 0$ \\
\hline $10 \cdot 5$ & $14100 \cdot 0$ & $6460 \cdot 0$ \\
\hline 11.0 & $14500 \cdot 0$ & $4170 \cdot 0$ \\
\hline
\end{tabular}

and $\rho_{\mathrm{p}}=1.0 \times 10^{3} \mathrm{~kg} / \mathrm{m}^{3}$. The real and imaginary parts of the complex modulus $G, G^{I}$ and $G^{H}$, respectively, are shown in Table 1 as functions of frequency $\omega$. To obtain values of $\omega$ which are not given by the table, a second order least-square curve fitting technique was used.

The unavailability of other "exact-theory" results made comparisons impossible, so that considerable additional care had to be taken in the actual computational process. Discretization errors are of the order of $h^{2}$, where $h$ is the distance between adjacent nodes. Studies were made on the value of some typical eigenvalues as a function of $h$ and, by noting the asymptotic values, an accurate mesh size could be chosen. The studies led to a choice of 82 elements, which gave accuracy in the $2-3 \%$ range. Round-off error was assessed by comparing some single and double precision runs, as described in the book by Conte and De Boor [14]. Discrepancies of no more than 3\% arose, which was felt to be satisfactory.

The wavenumber $q$ is in general complex, i.e., $q=q_{r}+i q_{i}$, and the relation between $\omega$ and $q$ constitutes a surface. For the sake of visualization, only plots of $\omega$ versus $q_{r}$ and $q_{i}$ 


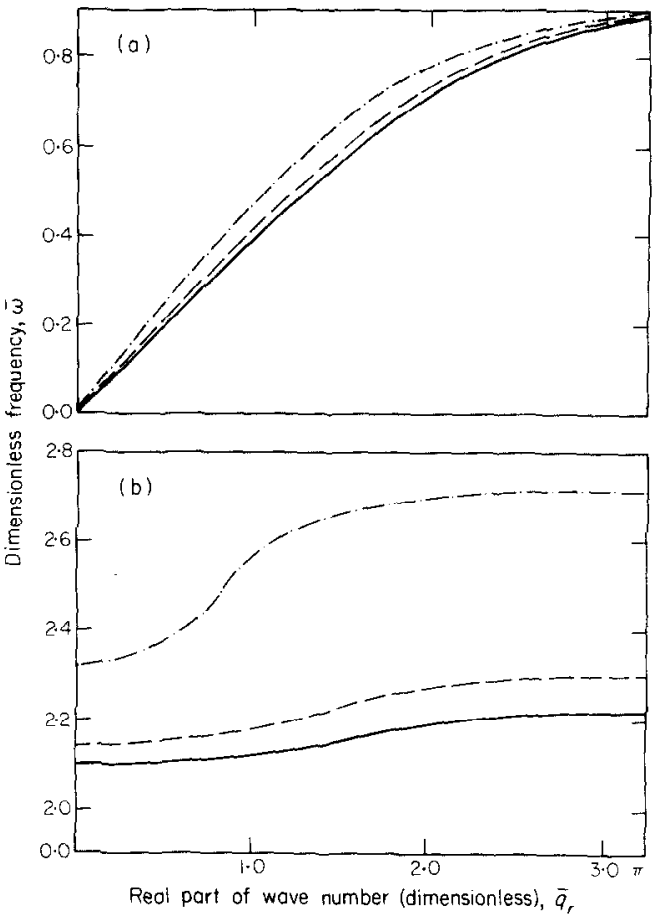

Figure 3. Dimensionless frequency versus real part of wavenumber. (a) First mode; (b) second mode. - $q_{1}=0.0 ;--, \bar{q}_{1}=0.15 ;--, \bar{q}_{1}=0.30$.

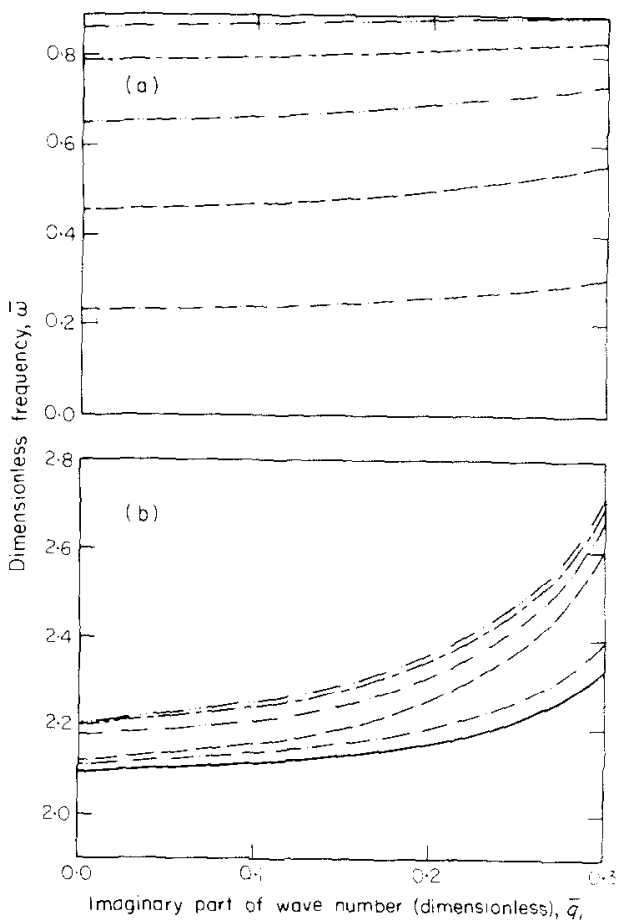

Figure 4. Dimensionless frequency versus imaginary part of wavenumber. (a) First mode; (b) second mode. 一, $\bar{q}_{r}=0.0 ;-\cdot, \bar{q}_{r}=0.6 ;--, \bar{q}_{r}=1 \cdot 2 ; \cdots,-, \bar{q}_{r}=1 \cdot 8 ;--,, \bar{q}_{r}=2.4 ;-\cdots, \bar{q}_{r}=\pi$. 
are presented. Results are given for the first two modes of propagation for the case $b=0.5 \mathrm{a}$. Figure 3 presents the dimensionless frequency $\bar{\omega}=\omega a \sqrt{\rho_{v} / K_{v}}$ versus dimensionless real wavenumber $\vec{q}_{r}=q_{r}$ a for several values of the dimensionless imaginary wavenumber $\bar{q}_{i}=q_{i} a$. It is seen that the curves rise with increasing $\bar{q}_{i}$, a feature that is much more pronounced for the second mode, as Figure 3(b) shows. The same phenomena can also be clearly seen in the plots of $\bar{\omega}$ versus $\bar{q}_{i}$ for several values of $\bar{q}_{r}$ given in Figure 4 . Note that the values of $\bar{\omega}$ associated with the second mode are orders of magnitude greater than those in the first mode. This implies the rapid damping of the higher modes, an important point to remember in the construction of approximate theories, since only low frequencies may be significant. Note further that attenuation increases with frequency, a trend also found by Sutherland [9] in related experimental work on fiber-reinforced quartz phenolic.

The figures also show that pass and no-pass bands exist. Actually they are a threedimensional zone between the surfaces of the first and second modes. Note too that the slopes of the $\bar{\omega}$ versus $\bar{q}_{r}$ curves at $\bar{q}_{r}=0, \pi$ are zero, except at the origin, and that the largest slope of the first mode is at the origin. These features also occur when the matrix material is elastic.

\section{REFERENCES}

1. G. J. DvoRaK (Chairman and Co-ordinator) 1978 Research Workshop on Mechanics of Composite Materials, 17-18 October 1978, Duke University, Durham, North Carolina.

2. R. A. SCoTt 1978 Shock and Vibration Digest 10, 25-41. Linear elastic wave propagation: an annotated bibliography: Part I.

3. R. A. SCOTT 1978 Shock and Vibration Digest 10, 11-39. Linear elastic wave propagation: an annotated bibliography: Part II.

4. R. J. Alfrey and R. A. ScotT 1978 Journal of Sound and Vibration 60, 239-250. A comparative study on transverse elastic wave propagation in laminates: subsonic case.

5. M. Stern, A. BEDFORD and C. H. YEW 1971 Journal of Applied Mechanics 38, 448-454. Wave propagation in viscoelastic laminates.

6. H. DemiraY and A. C. ERINGEN 1971 Journal of Sound and Vibration 55, 509-519. Wave propagation in viscoelastic composites reinforced by orthogonal fibers.

7. S. MUKHERJEE and E. H. LeE 1978 Computers and Structures 5, 279-285. Dispersion relations and mode shapes for waves in laminated viscoelastic composites by finite difference methods.

8. S. MUKHERJEE and E. H. LeE 1978 International Journal of Solids and Structures 14, 1-13. Dispersion relations and mode shapes for waves in laminated viscoelastic composites by variational methods.

9. H. J. SUTHERLAND 1975 Journal of the Acoustical Society of America 57, 870-875. Dispersion of acoustic waves by fiber-reinforced viscoelastic materials.

10. G. H. Golub, L. Jenning and W. H. YANG 1975 Journal of Computational Physics 17, 349-357. Waves in periodically structured media.

11. Y. C. Fung 1965 Foundations of Solid Mechanics. New Jersey: Prentice-Hall, Inc. 1976.

12. P. G. Ciarlet 1976 Numerical Analysis of the Finite Element Method. Les Presses De L'Universite de Montreal.

13. T.-C. MA 1979 Ph.D. Dissertation, Department of Applied Mechanics and Engineering Science, University of Michigan. Harmonic wave propagation in porous media and in fiber reinforced viscoelastic media.

14. S. D. CONTE and C. De BOOR 1965 Elementary Numerical Analysis: An Algorithmic Approach. New York: McGraw-Hill, Inc. 